\title{
Inflammatory peroxidases promote breast cancer progression in mice via regulation of the tumour microenvironment
}

\author{
VASILIOS PANAGOPOULOS ${ }^{1}$, DAMIEN A. LEACH $^{1}$, IRENE ZINONOS ${ }^{1}$, \\ VLADIMIR PONOMAREV ${ }^{2}$, GIOVANNI LICARI ${ }^{1}$, VASILIOS LIAPIS ${ }^{1}$, WENDY V. INGMAN $^{1}$, \\ PETER ANDERSON $^{3}$, MARK O. DeNICHILO ${ }^{1}$ and ANDREAS EVDOKIOU ${ }^{1}$ \\ ${ }^{1}$ Discipline of Surgery, Breast Cancer Research Unit, Basil Hetzel Institute and Centre for \\ Personalised Cancer Medicine, University of Adelaide, Adelaide, SA, Australia; ${ }^{2}$ Department of Radiology, \\ Memorial Sloan-Kettering Cancer Center, New York, NY, USA; ${ }^{3}$ Australian Craniofacial Unit, \\ Women's and Children's Health Network, Adelaide, SA, Australia
}

Received October 18, 2016; Accepted November 28, 2016

DOI: 10.3892/ijo.2017.3883

\begin{abstract}
Myeloperoxidase (MPO) and eosinophil peroxidase (EPO) are heme-containing enzymes, well known for their antimicrobial activity, are released in high quantities by infiltrating immune cells in breast cancer. However, the functional importance of their presence within the tumour microenvironment is unclear. We have recently described a new role for peroxidases as key regulators of fibroblast and endothelial cell functionality. In the present study, we investigate for the first time, the ability of peroxidases to promote breast cancer development and progression. Using the 4T1 syngeneic murine orthotopic breast cancer model, we examined whether increased levels of peroxidases in developing mammary tumours influences primary tumour growth and metastasis. We showed that MPO and EPO stimulation increased mammary tumour growth and enhanced lung metastases, effects that were associated with reduced tumour necrosis, increased collagen deposition and neo-vascularisation within the primary tumour. In vitro, peroxidase treatment, robustly stimulated human mammary fibroblast migration
\end{abstract}

Correspondence to: Professor Andreas Evdokiou, Basil Hetzel Research Institute, The Queen Elizabeth Hospital, 28 Woodville Road, Woodville, SA 5011, Australia

E-mail: andreas.evdokiou@adelaide.edu.au

Abbreviations: AA, ascorbic acid; ANOVA, analysis of variance; BLI, bioluminescence imaging; COX-2, cyclooxygenase-2; DAPI, 4',6-diamidino-2-phenylindole; DMEM, Dulbecco's modified Eagle's medium; ECM, extracellular matrix; ELISA, enzyme linked immunosorbent assay; EPO, eosinophil peroxidase; FBS, fetal bovine serum; G, gauge; H\&E, hematoxylin and eosin; i.p, intraperitoneal; MMP, matrix metalloproteinase; MPO, myeloperoxidase; PBS, phosphate-buffered saline; PET, polyethylene terephthalate; RPMI, Roswell Park Memorial Institute; SEM, standard error of the mean; $\alpha$-SMA, alpha-smooth muscle actin

Key words: myeloperoxidase, eosinophil peroxidase, tumour inflammation, stromal microenvironment, breast cancer and collagen type I and type VI secretion. Mechanistically, peroxidases induced the transcription of pro-tumorigenic and metastatic MMP1, MMP3 and COX-2 genes. Taken together, these findings identify peroxidases as key contributors to cancer progression by augmenting pro-tumorigenic collagen production and angiogenesis. Importantly, this identifies inflammatory peroxidases as therapeutic targets in breast cancer therapy.

\section{Introduction}

It is well-established that chronic inflammation is a critical component of tumour development and progression $(1,2)$. However, many of the molecular and cellular factors involved remain obscure. Infiltrating neutrophils and eosinophils have been observed in most human cancers and are often associated with poor survival (3-5). Recently, neutrophils have gained considerable attention as important players in cancer progression because of their role in ECM remodeling, tumour angiogenesis and metastasis (6-8). Moreover, Chen et al (5) revealed that an elevated peripheral neutrophil/lymphocyte ratio was associated with poor survival in breast cancer.

Within the tumour microenvironment, the accumulation of neutrophils is reported within the invasive front of tumours $(9,10)$, while eosinophils abundantly accumulate near blood vessels and infiltrate hypoxic regions $(11,12)$, where they are activated to release an arsenal of factors from their cytoplasmic granules. A major component of these released factors are myeloperoxidase (MPO) and eosinophil peroxidase (EPO), which are released by infiltrating neutrophils and eosinophils, respectively. Importantly, these enzymes are abundantly deposited in breast cancer $(4,13)$. However, to date, no distinct role has been attributed to their presence within the tumour microenvironment. We have recently reported a new biological role for peroxidase enzymes as key regulators of fibroblasts and endothelial cell functionality, driving migration, collagen biosynthesis and blood vessel development $(14,15)$, processes which are major contributors of cancer development and metastasis (16). 
In the present study, we investigated for the first time the pro-tumorigenic and pro-metastatic potential of MPO and EPO using the 4T1 syngeneic breast cancer mouse model and characterized the molecular mechanisms involved. In vitro, peroxidase treatment stimulated primary human mammary fibroblast migration, collagen type I and VI protein secretion, and modified ECM architecture. In vivo, peroxidases enhanced mammary tumour growth and progression. Taken together, our findings identify peroxidase enzymes as key mediators of tumour development and progression, and suggest that peroxidase inhibitors may have therapeutic potential for the treatment of breast cancer and metastasis.

\section{Materials and methods}

Reagents. Native human eosinophil peroxidase (EPO) was obtained from Cell Sciences (Canton, MA, USA) and GenWay Biotech Inc. (San Diego, CA, USA). Recombinant human myeloperoxidase (MPO) was purchased from R\&D Systems (Minneapolis, MN, USA).

Animals. Five week-old female BALB/c mice (Institute of Medical and Veterinary Services Division, Gilles Plains, SA, Australia) were acclimatized to the animal housing facility for a minimum period of 1 week prior to the commencement of experimentation. All mice were housed under pathogen free conditions and all experimental procedures on animals were carried out with strict adherence to the rules and guidelines for the ethical use of animals in research and were approved by the Animal Ethics Committees of the University of Adelaide and the Institute of Medical and Veterinary Science, Adelaide, SA, Australia.

Mammary fat pad injection of breast cancer cells. 4T1 mouse breast cancer cells were cultured in RPMI-1640 media, supplemented with $2 \mathrm{mM}$ glutamine, $100 \mathrm{IU} / \mathrm{ml}$ penicillin, $160 \mu \mathrm{g} / \mathrm{ml}$ gentamicin, HEPES (20 mM) and $10 \%$ fetal bovine serum (FBS; Biosciences, Sydney NSW, Australia) in a 5\% $\mathrm{CO}_{2}$-containing humidified atmosphere, until they reached $70-80 \%$ confluent. Adherent cells were removed from flasks with $0.5 \%$ trypsin/EDTA and resuspended in phosphate-buffered saline (PBS) at $0.5 \times 10^{5}$ cells $/ 10 \mu \mathrm{l}$ and kept on ice in an Eppendorf tube. An equal volume of Matrigel $^{\mathrm{TM}}-\mathrm{HC}$ (BD Biosciences, Bedford, MA, USA) was added to the cells and resuspended. Seven-week old mice were divided into 3 groups $(n=7)$ and anaesthetized by isoflurane (Faulding Pharmaceuticals, Adelaide, Australia). The mammary fat pad area of the mice was wiped with ethanol and the skin was lifted over the left outermost nipple. Finally, a 25-G needle was inserted and $20 \mu \mathrm{l}$ of cells were injected into the mammary fat pad. Mice were allowed to recover under the heat lamp before being transferred into cages. Mice were humanely sacrificed 23 days after the cancer cell transplantation, due to high tumour load and the lungs were removed for assessment of tumour burden.

In vivo bioluminescent imaging (BLI). Non-invasive, whole body imaging to monitor luciferase-expressing murine 4T1 mammary carcinoma cells in mice was performed weekly using the IVIS Spectrum ${ }^{\mathrm{TM}}$ imaging system (Xenogen Corp.,
Alameda, CA, USA). Mice were injected into the intraperitoneal (i.p.) space with $100 \mu \mathrm{l}$ of the D-Luciferin solution at final dose of $3 \mathrm{mg} / 20 \mathrm{~g}$ mouse body weight (Xenogen Corp.) and then gas-anaesthetized with isoflurane (Faulding Pharmaceuticals). Images were acquired for 0.5-30 sec (representative images are shown at $1 \mathrm{sec}$ ) from the front angle and the photon emission transmitted from mice was captured and quantitated in photons/sec using Living Image software (version 4.2).

Histochemistry analysis. Standard hematoxylin and eosin (H\&E) staining of paraffin embedded tissue was used for histological examination of primary tumours. Stained sections were examined and photographed on a Nikon Eclipse 90i microscope. Tumour necrosis was assayed on H\&E whole tumour cross-section and quantified by ImageJ software $(\mathrm{NIH}$, Bethesda, MD, USA) as previously described (17). Briefly, $\mathrm{H} \& \mathrm{E}$ histological slides of whole tumour cross-sections were digitized, where areas of tumour borders and necrosis were assessed and highlighted as a separate pixelated area. The area of tumour necrosis was then quantified as a percentage of total tumour area. For immunohistochemistry, sections ( $6 \mu \mathrm{m}$ thick) of 4T1 tumours were probed with primary antibodies; rabbit anti-human collagen type I polyclonal; rabbit anti-human collagen type VI polyclonal (Rockland Immunochemicals, Inc., Pottstown, PA, USA), mouse anti-human $\alpha$-SMA (Sigma-Aldrich, St. Louis, MO, USA), rabbit anti-human CD31 (Abcam, Cambridge, UK) as previously described (14). Quantitative analysis of the degree of positive staining was performed by digitized image analysis with ImageJ software as previously described (18). Briefly, the image of the tissue area was converted to grey scale and the threshold level of staining was set for each image and measured and expressed as a percentage of the total tissue surface.

Transwell migration assay. Mammary fibroblast cell migration was determined using the 24-well Transwell plate (BD Falcon FluoroBlok $^{\mathrm{TM}}$ ) system with $8.0 \mu \mathrm{m}$ pore PET membranes. Fibroblasts were starved in serum-free Dulbecco's modified Eagle's medium (DMEM) overnight, then seeded ( $1 \times 10^{5}$ cells) in $100 \mu \mathrm{l}$ of serum-free DMEM into the upper wells and incubated at $37^{\circ} \mathrm{C}$ in $5 \% \mathrm{CO}_{2}$ for 30 min to allow cell adhesion. Lower chambers were then filled with $700 \mu \mathrm{l}$ of serum-free DMEM with no further supplementation as the negative control, or supplementation with TGF- $\beta$ (10 ng/ml) serving as the positive control, or with the various peroxidase proteins. Cells were allowed to migrate for $18 \mathrm{~h}$. Migrated cells were fixed in 6:1 ethanol/acetic acid for $10 \mathrm{~min}$, stained with DAPI $(1 \mu \mathrm{g} / \mathrm{ml})$, and then photographed and quantified on a fluorescent inverted microscope (Axio Observer Z1; Carl Zeiss AG, Oberkochen, Germany).

Collagen enzyme-linked immunosorbent assay (ELISA). To evaluate the effect of MPO and EPO on collagen production, human mammary fibroblasts were seeded into 96-well plates (Nunc, Roskilde, Denmark) at a density of $1.2 \times 10^{4}$ cells/well and cultured for 5 days in DMEM/10\% FBS until reaching confluence. Cells were starved overnight in serum-free DMEM and then stimulated for an additional $72 \mathrm{~h}$ in serum-free DMEM containing either ascorbic acid 2-phosphate at $10 \mu \mathrm{mol} / 1$ (Wako Chemical Industries, Ltd., Osaka, Japan) as a positive control, 
or with the peroxidase proteins in the absence of ascorbic acid supplementation. At the end of the 72-h treatment period, fibroblast-conditioned media were collected for measurement of secreted soluble type I and VI collagen by ELISA. Cell viability/ growth was then assessed using the AlamarBlue ${ }^{\mathrm{TM}}$ fluorescent dye assay (Invitrogen Life Technologies, Melbourne, Australia) as per manufacturer's instructions. The amount of soluble type I and VI collagen in cell-conditioned medium was measured by a direct coat ELISA method using standard curves constructed from purified type I and VI human placental collagen (BD Biosciences) as previously described (14).

$3 D$ matrix production. To assess the effect of MPO and EPO on 3D collagen formation, 3D collagen matrices were generated by stimulated human mammary fibroblasts as previously described (19). Briefly, 24-well plates were treated with $0.2 \%$ gelatin in PBS overnight at $4^{\circ} \mathrm{C}$, then cross-linked with paraformaldehyde and washed multiple times with PBS. Human mammary fibroblasts were seeded into plates and grown to confluency. When cells reached $100 \%$ confluency, platting media were replaced with reduced serum (2\% FBS) DMEM supplemented with $1 \mu \mathrm{g} / \mathrm{ml}$ of MPO or EPO. Ascorbic acid 2-phosphate at $10 \mu \mathrm{M}$ and $2 \%$ FBS DMEM were used as positive and vehicle controls, respectively. Treatment media were replaced every second day for six days. Fibroblasts were then removed using cell lysis buffer, and the remaining ECM was washed multiple times with PBS prior to use in adhesion, invasion and immunofluorescence assays.

Immunofluorescence assays. Human mammary fibroblasts were seeded onto circular glass coverslips where 3D collagen matrices were generated. Matrices were blocked with goat serum in PBS Triton-X, before washing and immune-labeled overnight with antibodies against collagen type I (Rocklands Immunochemicals). Labeled proteins were detected using Alexa Fluor ${ }^{\circledR} 488$ goat anti-mouse IgG (Molecular Probes/ Thermo Fisher Scientific Australia Pty Ltd., Scoresby VIC, Australia). Fluorescence was captured using a laser confocal microscope (Zeiss LSM 700; Carl Zeiss AG).

4 T1 mammary carcinoma adhesion assay. Using 3D-ECM matrices created from fibroblasts, adherence of 4T1 cancer cells was assessed via adhesion assays previously described (20). Briefly, $2 \times 10^{4}$ cells were applied to $3 \mathrm{D}$-ECMs in 24 -well plates and were allowed to adhere for $4 \mathrm{~h}$. Cells were then gently washed with PBS before the cell measurement via AlamarBlue $^{\mathrm{TM}}$ assay as per manufacturer's instructions.

4T1 mammary carcinoma invasion assay. Three-dimensional (3D) ECM invasion assays were adapted from a previously described study (21). Mammary fibroblast derived 3D-ECM matrices were created in the upper chamber of a 24-well Transwell plate (BD Falcon FluoroBlok ${ }^{\mathrm{TM}}$ ) system with $8.0 \mu \mathrm{m}$ pore PET membranes. 4T1 cells were then seeded ( $1 \times 10^{5}$ cells) in $100 \mu \mathrm{l}$ of serum-free RPMI into the upper wells and incubated at $37^{\circ} \mathrm{C}$ in $5 \% \mathrm{CO}_{2}$ for $30 \mathrm{~min}$ to allow cell adhesion. Lower chambers were filled with $700 \mu \mathrm{l}$ of reduced serum $(2 \%$ FBS) RPMI as a chemo-attractant stimulus for $24 \mathrm{~h}$. Migrated cells on the opposite side of the Transwell membrane were fixed in 6:1 ethanol/acetic acid for $10 \mathrm{~min}$, stained with DAPI
(1 $\mu \mathrm{g} / \mathrm{ml})$, and then photographed and quantified on a fluorescent inverted microscope (Axio Observer Z1; Carl Zeiss AG).

$4 T 1$ mammary carcinoma proliferation assay. Proliferation of sub-confluent 4T1 cultures was assessed by alamarBlue, as previously described (22). Briefly, 4T1 $\left(1 \times 10^{4}\right)$ cells were cultured in 96-well plates in $100 \mu \mathrm{l}$ in $2 \%$ FBS RPMI media. Cells were treated with $100 \mu \mathrm{l}$ of increasing concentrations of MPO and EPO $(0.31-5 \mu \mathrm{g} / \mathrm{ml})$ at the time of cell plating and cultured for $48 \mathrm{~h}$. FBS RPMI growth media (10\%) served as the positive control. The fluorescence was measured and quantified at wavelengths of $530 \mathrm{~nm}$ excitation and $595 \mathrm{~nm}$ emission using FLUOstar Optima microplate reader (BMG Labtech, Ortenberg, Germany).

RNA isolation and quantitative real-time PCR. To evaluate the effect on human mammary fibroblast and 4T1 mammary carcinoma gene regulation by MPO and EPO $(2 \mu \mathrm{g} / \mathrm{ml})$, RT-PCR was performed. RNA isolation and cDNA synthesis was performed as previously described (14). Messenger RNA expression of specific genes was identified using real-time RT-PCR using SYBR-Green Fluor qPCR Mastermix (Qiagen) in a CFX96 real-time system (Bio-Rad Laboratories) as previously described (23). The primer combinations used are as follows: cyclooxygenase-2 (COX-2): forward, 5'-CCTGTG CCTGATGATTGC-3' and reverse, 5'-CTGATGCGTGAAG TGCTG-3'; matrix metalloproteinase 1 (MMP1): forward, 5'-GACGTTCCCAAAAATC-3' and reverse, 5'-GCTAGAAG GGATTG-3'; matrix metalloproteinase 3 (MMP3): forward, 5'-AGAGGCATCCACACC-3' and reverse, 5'-CTGGCTCCA TGGAATTTCTCTTC-3'. Expression values were normalized to the housekeeping gene glyceraldehyde-3-phosphate dehydrogenase (GAPDH): forward, 5'-ACCCAGAAGACTCTGTG GATGG-3' and reverse, 5'-TCAGTGAGCTTCCCGTTCAG-3'

Data analysis and statistical analysis. Data points derived from experiments are reported as the mean \pm standard error of the mean (SEM). Analysis of variance to determine significant difference between samples was performed using the paired Student's t-test, and one way ANOVA followed by multiple comparison test (Dunnett's Method) where indicated, using SigmaPlot 2011 (version 12.0; Systat Software, Inc., San Jose, CA, USA).

\section{Results}

Peroxidases enhance primary tumour growth in vivo. To evaluate the role of MPO and EPO in mammary tumour growth and metastasis, we used a bioluminescent, luciferase labeled 4T1 mouse mammary carcinoma cell line, and the subsequent orthotopic 4T1 tumour metastasis model in BALB/c mice. 4T1 cells were inoculated into the 4th mammary fat pad of $\mathrm{BALB} / \mathrm{c}$ mice and allowed one week for tumour establishment before once weekly intratumoral injections of 5- $\mu \mathrm{g}$ doses of MPO or EPO, with PBS serving as the vehicle control. Tumour growth was monitored weekly with non-invasive bioluminescence imaging (BLI) (Fig. 1A), and quantified via luciferase intensity (photons/sec) (Fig. 1B), which showed that both MPO and EPO treatment increased primary tumour growth (MPO, $\mathrm{P}=0.05$ ), over 23 days, as compared to the vehicle control. 

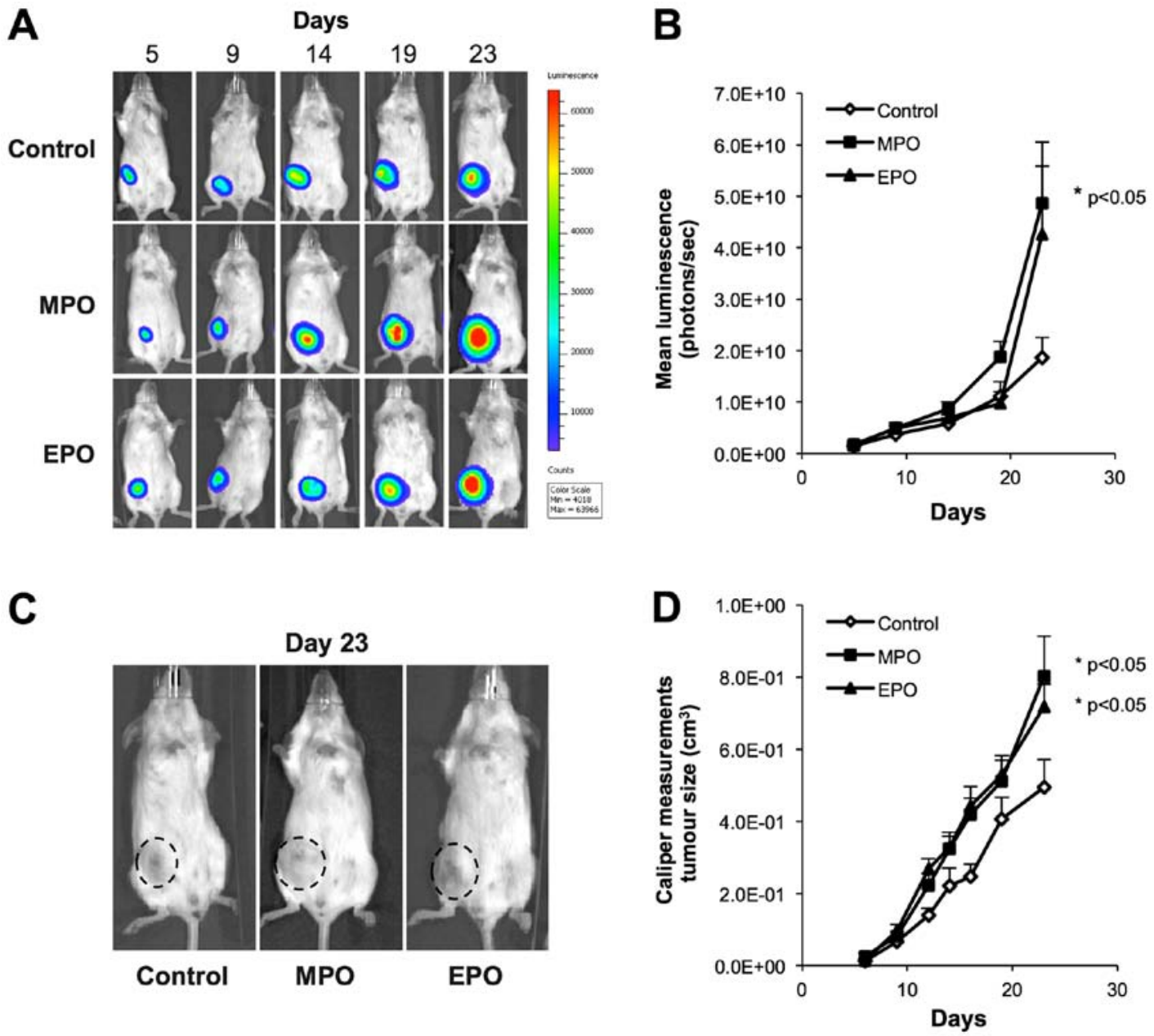

Figure 1. Effect of increased levels of peroxidases on tumour growth. (A) Seven-week old female BALB/c mice were injected with $0.5 \times 10^{5} 4 \mathrm{~T} 1$ mammary carcinoma cells orthotopically into the 4th mammary fat pad. Mice were imaged weekly using the Xenogen IVIS-Spectrum bioluminescence imaging system. Representative whole body BLI images of a single animal from each group $(n=7)$ during the course of the experiments are shown. All animals were humanely sacrificed on day 23 for ethical reasons, due to high tumour load in the treatment groups. (B) The line graph represents the average tumour signal over time measured as mean photon counts per sec. (C) Representative images of tumours taken at day 23 where tumour growth was also monitored using caliper measurements. (D) Quantification of caliper measurements of tumour size $\left(\mathrm{cm}^{3}\right)$ taken twice weekly throughout the course of the study. Results are shown as the mean \pm SEM. Statistical significance was calculated by ANOVA $\left({ }^{*} \mathrm{P}<0.05\right)$.

Tumour burden measured also by calipers and expressed as a function of volume in $\mathrm{mm}^{3}$ confirmed the BLI $(\mathrm{P}<0.05$; Fig. 1C and D).

Histological examinations of the 4T1 tumours after 23 days (Fig. 2A) showed that tumour necrosis was significantly decreased by MPO and EPO treatment. Representative images of $\mathrm{H} \& \mathrm{E}$ staining clearly show the inherent necrotic and avascular regions within the PBS treated 4T1 primary tumour in the control mice. In contrast, treatment with either peroxidase, show diminished regions of necrotic areas that were associated with the presence of viable tumour cells. Histological staining and subsequent quantitative image analysis of tumour sections with antibodies to CD31, $\alpha$-SMA and collagen types I and VI, identified and increase in vascularization, myofibroblasts and ECM deposition, respectively (Fig. 2B).

Peroxidases enhance lung metastasis. Mammary carcinoma 4T1 cells spontaneously metastasize to the lungs from the mammary fat pad (24). Histological assessment of excised lungs stained with $H \& E$ confirmed the presence of lung metastasis indicating an increase in macrometastases in the lungs of animals treated with peroxidases (Fig. 3A). Ex vivo BLI quantification of excised lungs from animals in each group confirmed these findings as a function of photon counts per sec. Both MPO and EPO treatment groups revealed an increase in the intensity (photons/sec) representative of increased spontaneous lung metastasis (MPO, $\mathrm{P}<0.05$; EPO, $\mathrm{P}=0.12$ ) after 23 days relative to vehicle control (Fig. 3B). Thus, our data indicate that an increase in peroxidase levels within the primary tumour has the potential to promote metastatic spread to the lungs in vivo.

Peroxidases promote pro-fibrotic effects in primary human mammary fibroblasts in vitro. The migratory ability of fibroblasts within the tumour microenvironment plays a central role in tumour growth and is causatively associated with increased metastatic behaviour of cancer cells (25). To determine the effect of peroxidases on primary human mammary fibroblasts, we measured cellular movement toward MPO and EPO using a Transwell migration assay. After $24 \mathrm{~h}$ in serum-free conditions, MPO and EPO $(5 \mu \mathrm{g} / \mathrm{ml})$ significantly increased mammary fibroblast migration by 2 - and 4-fold, respectively, 


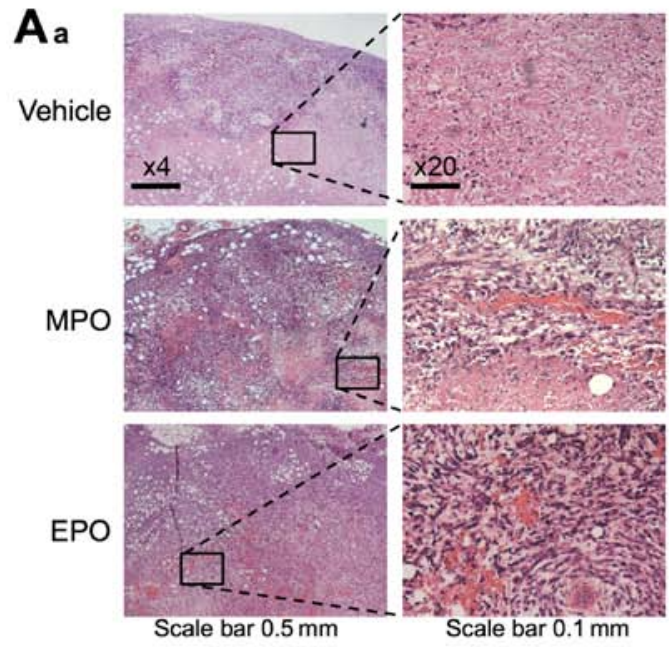

b

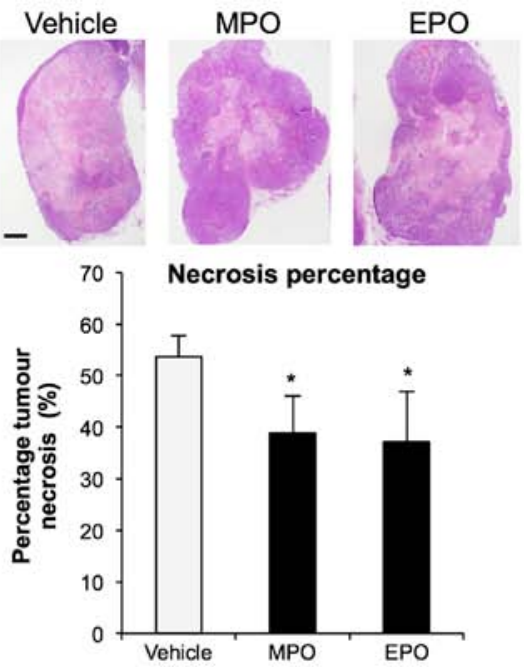

$\mathbf{B}_{\mathbf{a}}$
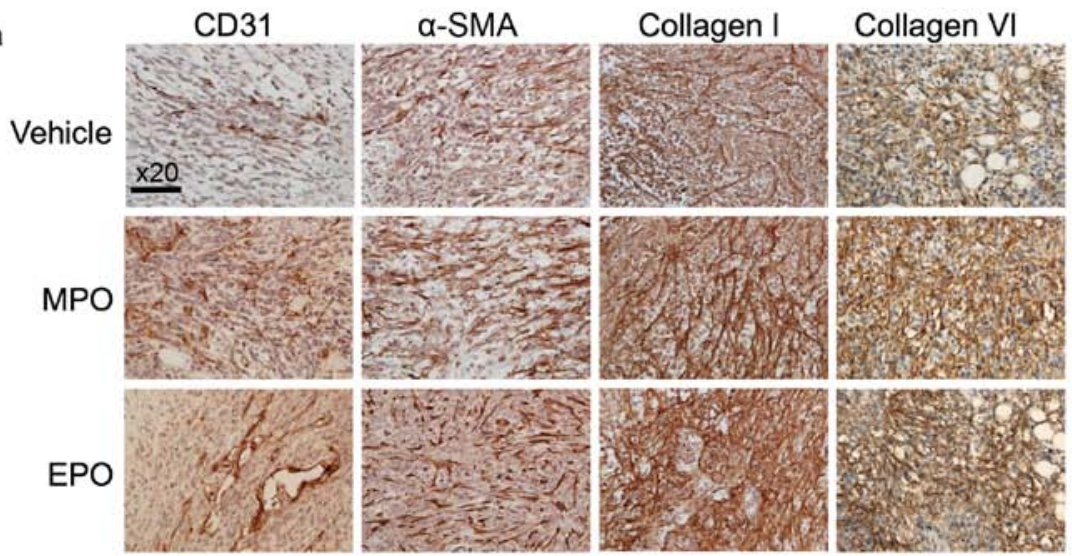

b
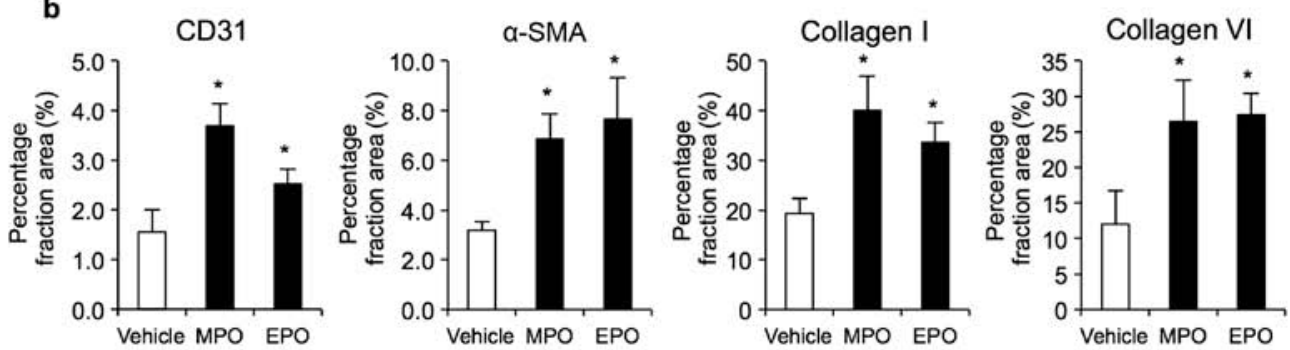

Figure 2. Histological assessment of peroxidase effects within 4T1 tumours. (A) Representative images of 4T1 tumour sections at $\mathrm{x} 4$ and $\mathrm{x} 20$ magnification after 23 days treated with either saline (vehicle) or $5 \mu \mathrm{g}$ weekly doses of MPO or EPO stained with hematoxylin and eosin (H\&E) for morphology within the necrotic regions (a) and whole tumour images (Scale bar, $2 \mathrm{~mm}$ ) and quantification of the average percentage tumour necrotic area from each group ( $\mathrm{n}=7$ ) using ImageJ software (b). (B) Specific immunostaining was conducted on 4 mice per group showing the distribution of (a) CD31, $\alpha$-SMA and collagen types I and VI within the necrotic regions of the tumour. (b) Quantitative image analysis of the fraction area stained positive for CD31, $\alpha$ SMA and collagen types I and VI. Each value indicates the mean \pm SEM of images taken within the necrotic area. Statistical significance was calculated by the Student's $t$-test ("P<0.05). Scale bars at $\mathrm{x} 4$ magnification, $500 \mu \mathrm{m}$ and at $\times 20$ magnification, $100 \mu \mathrm{m}$.

when compared to vehicle control $(\mathrm{P}<0.05$; Fig. $4 \mathrm{~A})$. The stimulation of primary human mammary fibroblasts with increasing concentrations of MPO and EPO in the absence of ascorbic acid (AA) demonstrated a dose-dependent increase in collagen type I (Fig. 4B) and type VI (Fig. 4C) up to 2and 1.6-fold, respectively $(\mathrm{P}<0.0001, \mathrm{P}<0.001$ and $\mathrm{P}<0.05)$. At these peroxidase concentrations, there was no impact on the proliferation and/or viability of the confluent mammary fibroblasts. To determine the potential for other mechanisms by which peroxidases activate fibroblasts, we next investigated the transcriptional regulation of the key genes implicated in promoting a motile phenotype (Fig. 4D) including COX-2 and the matrix modifying genes MMP1 and MMP3 via RT-PCR (26). Analysis of mRNA expression showed that MPO and EPO increased COX-2 expression 2.5- and 3.7-fold, MMP1 expression 2- and 2.9-fold, and MMP3 expression 1.8and 4.2-fold, respectively, when compared to vehicle control $(\mathrm{P}<0.0001, \mathrm{P}<0.001$ and $\mathrm{P}<0.05)$.

Peroxidases promote a functional pro-tumorigenic ECM in vitro. Collagen fiber alignment plays a critical role in tumour progression (27). Immunofluorescent detection of collagen type I orientation within cell derived 3D collagen matrices (Fig. 5A) showed that MPO (MPO-ECM) and EPO 


\section{B Pulmonary metastasis bioluminescence}
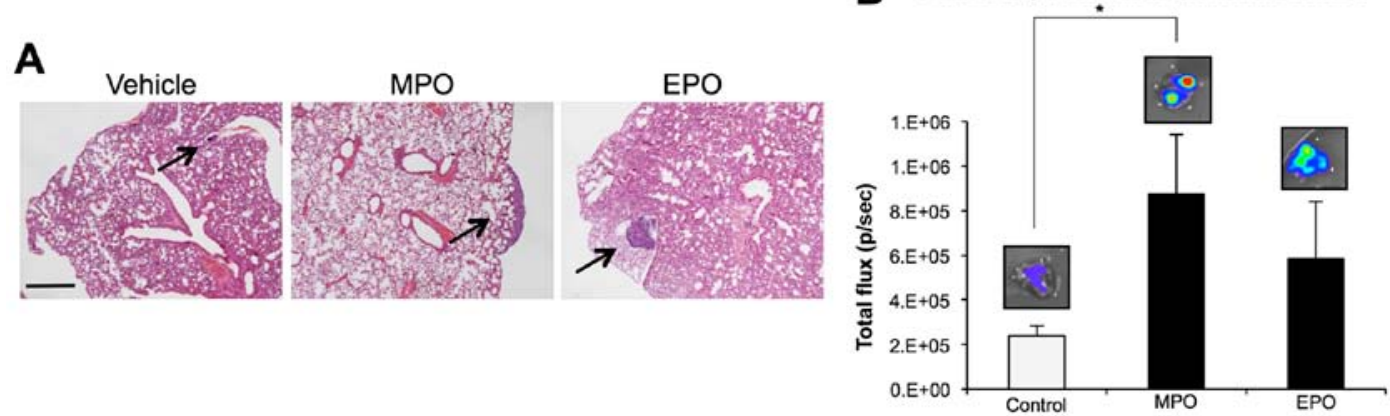

Figure 3. Effects of peroxidases on pulmonary metastasis. (A) Representative histological sections of lungs from each group stained with H\&E, arrows indicate the presence of macrometastasis, with showing an increase of tumour burden within the lungs of peroxidase treated mice. (B) Quantification of bioluminescent tumour signal in the lungs measured as average photon counts per second, with representative BLI images from each treatment group inoculated with 4T1 cells after 23 days. Results are shown as the mean \pm SEM. Statistical significance was calculated by ANOVA ("P<0.05). Scale bar, $500 \mu \mathrm{m}$.

A Mammary fibroblast migration after $24 \mathrm{~h}$

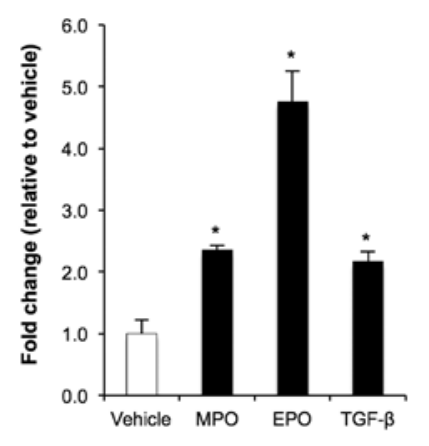

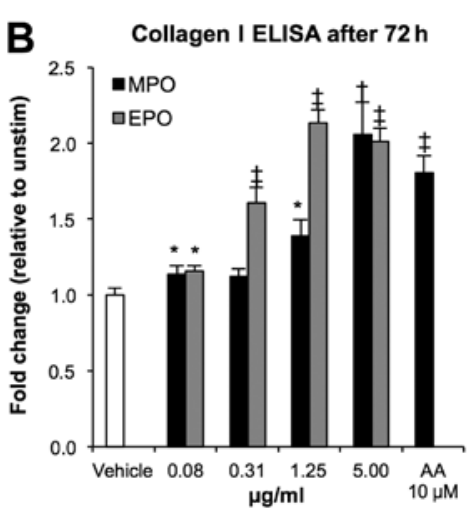

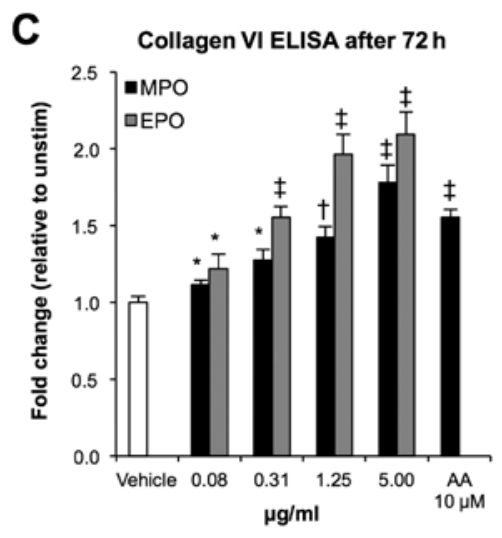

$\mathbf{D}_{\mathrm{a}}$

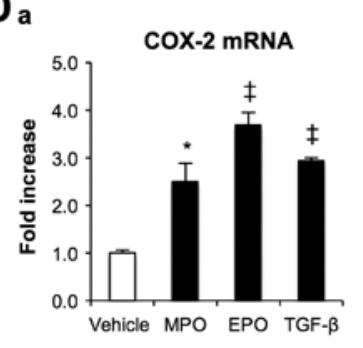

b

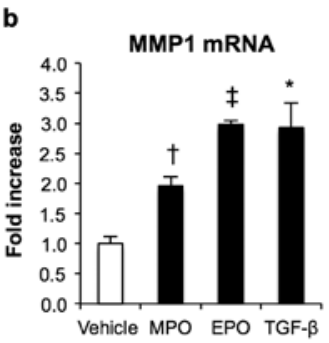

c

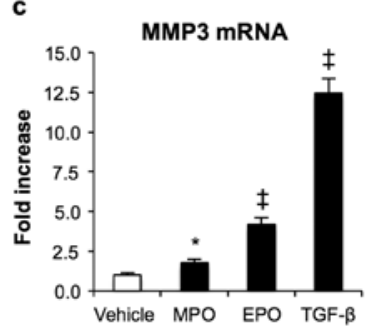

Figure 4. Peroxidases promote profibrotic effects in human mammary fibroblasts in vitro. (A) Transwell migration assay. Human mammary fibroblasts (5x104) were placed onto the membrane well of a Transwell insert in the presence of treatment medium in the lower chamber. Cells treated with serum-free culture media served as the vehicle control, TGF- $\beta(10 \mathrm{ng} / \mathrm{ml})$ served as the positive control, while MPO and EPO $(5 \mu \mathrm{g} / \mathrm{ml})$ were used as test. Migrated cells were stained with DAPI after $24 \mathrm{~h}$ and were counted from five fields of view in triplicate wells. Enzyme-linked immunosorbant assay detection of soluble collagen type I (B), and type VI (C) in primary human mammary fibroblasts conditioned media after $72 \mathrm{~h}$ of stimulation with MPO and EPO at the doses indicated. Ascorbic acid 2-phosphate (AA) at $10 \mu \mathrm{M}$ served as the positive control, whereas cells treated with serum-free culture media (DMEM) alone served as the vehicle control. The levels of collagen are expressed as fold change compared to vehicle control. (D) RT-qPCR analysis of mRNA from an independent set of triplicate human mammary fibroblast samples, stimulated with MPO or EPO $(0.5 \mu \mathrm{g} / \mathrm{ml})$ after $48 \mathrm{~h}$ for gene expression of (a) COX-2, (b) MMP1 and (c) MMP3. Data were normalized to the housekeeping gene GAPDH and expressed as fold change compared to vehicle control. Representative data are shown as the mean \pm SEM performed in quadruplicate of two independent experiments. Statistical significance was calculated by the Student's $t$-test ( ${ }^{\ddagger} \mathrm{P}<0.0001$, $\left.{ }^{\dagger} \mathrm{P}<0.001,{ }^{*} \mathrm{P}<0.05\right)$. 


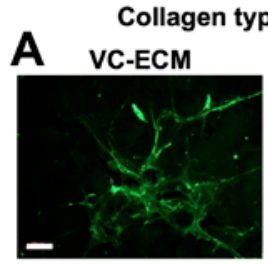

Collagen type I 3D-ECM
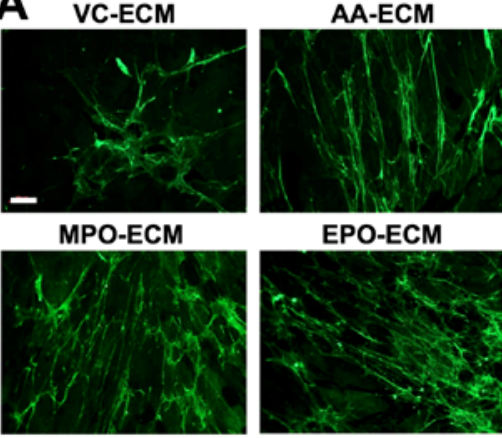

EPO-ECM
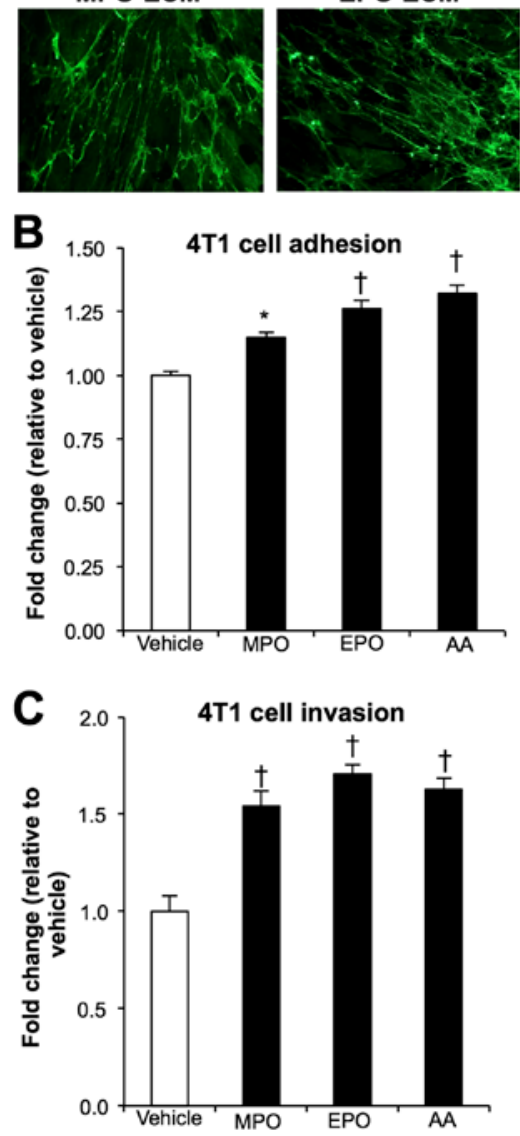

Figure 5. Peroxidases promote a functional pro-tumorigenic ECM. (A) Immunofluorescent detection of collagen type I fibers created from human mammary fibroblasts treated with $1 \mu \mathrm{g} / \mathrm{ml}$ of MPO (MPO-ECM) or EPO (EPO-ECM) after 6 days of culture. AA (AA-ECM) at $10 \mu \mathrm{M}$ served as the positive control, whereas serum reduced culture media (2\% FBS DMEM) served as the vehicle control (VC-ECM). (B) Adhesion assay. 4T1 $\left(5 \times 10^{4}\right)$ cancer cells were seeded onto MPO or EPO $(1 \mu \mathrm{g} / \mathrm{ml})$ stimulated ECM generated by human mammary fibroblasts after 6 days of culture for $4 \mathrm{~h}$. ECM generated by AA at $10 \mu \mathrm{M}$ served as the positive control, whereas serum reduced culture media (2\% FBS DMEM) served as the vehicle control. 4T cells were then washed off $2 X$ with PBS and remaining adhered cells were measured using AlamarBlue ${ }^{\mathrm{TM}}$ dye assay. (C) 4T1 $\left(1 \times 10^{5}\right)$ cancer cells were placed onto the membrane well of a Transwell insert coated with MPO or EPO $(1 \mu \mathrm{g} / \mathrm{ml})$ stimulated ECM generated by human mammary fibroblasts after 6 days of culture. ECM generated by AA at $10 \mu \mathrm{M}$ served as the positive control, whereas serum reduced culture media (2\% FBS DMEM) served as the vehicle control. Base culture media were present in all the lower chambers. Invaded cells on the opposite side of the membrane were stained with DAPI after $24 \mathrm{~h}$ and were counted from five fields of view in triplicate wells. Results are represented as fold change compared to vehicle \pm SEM of three independent experiments. Statistical significance was calculated by the Student's t-test $\left({ }^{\top} \mathrm{P}<0.001,{ }^{*} \mathrm{P}<0.05\right)$. Scale bar, $500 \mu \mathrm{m}$.

(EPO-ECM) stimulation produced a matrix of parallel and/or linearized collagen fibers comparable to the AA (AA-ECM) positive control. In contrast, the unstimulated vehicle control matrices (VC-ECM) display a mesh-like and disorganized organization of random collagen fibers.
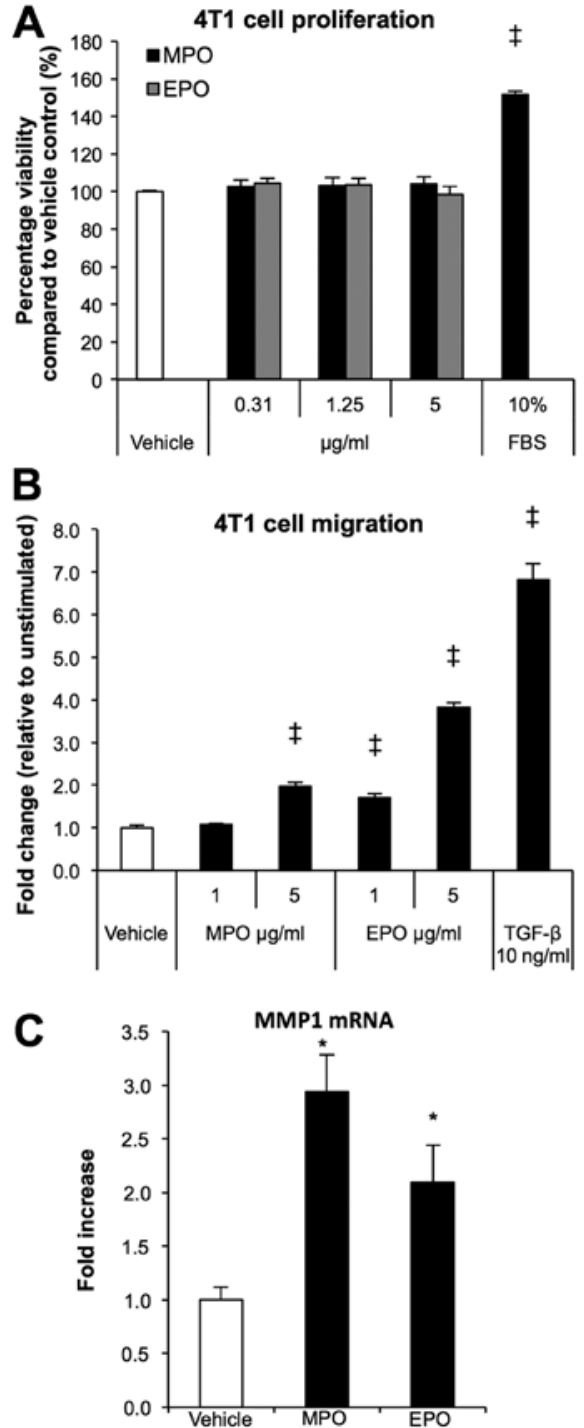

Figure 6. Effects of peroxidases on murine 4T1 carcinoma cells. (A) Proliferation assay. $4 \mathrm{~T} 1\left(1 \times 10^{4}\right)$ cells were plated and stimulated with MPO or EPO at the doses indicated for $48 \mathrm{~h}$. AA at $10 \mu \mathrm{M}$ served as the positive control, whereas base culture media (2\% FBS DMEM) served as the vehicle control and proliferation was measured as percentage of viability using AlamarBlue ${ }^{\mathrm{TM}}$ dye assay. (B) Transwell migration assay. 4T1 $\left(1 \times 10^{5}\right)$ cells were placed onto the membrane well of a Transwell insert in the presence of treatment medium in the lower chamber. Cells treated with base culture media served as the vehicle control, TGF- $\beta(10 \mathrm{ng} / \mathrm{ml})$ served as the positive control, while MPO and EPO (5 and $1 \mu \mathrm{g} / \mathrm{ml})$ were used as test. Migrated cells were stained with DAPI after $24 \mathrm{~h}$ and were counted from five fields of view in triplicate wells and shown as fold change compared to vehicle control. (C) RT-qPCR analysis of mRNA from an independent set of triplicate 4T1 samples, stimulated with MPO or EPO $(0.5 \mu \mathrm{g} / \mathrm{ml})$ after $48 \mathrm{~h}$. Data was normalized to the housekeeping gene GAPDH and expressed as fold change compared to vehicle control. Representative data are shown as the mean \pm SEM performed in quadruplicate of two independent experiments. Statistical significance was calculated by the Student's t-test $\left({ }^{*} \mathrm{P}<0.0001,{ }^{*} \mathrm{P}<0.05\right)$.

To determine whether this increase in the alignment of collagenous matrix affects tumour cell behaviour, we next tested the adherent properties of the different ECM by measuring 4T1 tumour cell adhesion to the matrices produced under different conditions. After $4 \mathrm{~h}$ of co-incubation, MPO-ECM and EPO-ECM significantly increased 4T1 adhesion up to $15 \%(\mathrm{P}<0.01)$ and $25 \%(\mathrm{P}<0.001)$, respectively, when compared to VC-ECM. The response was similar to 
that achieved by the AA-ECM (Fig. 5B). Collagen alignment facilitates invasion, thus, to further understand how the reorganization of aligned collagen fibers interacts with tumour cells, we tested the effects of the peroxidase-modified matrices on the $4 \mathrm{~T} 1$ cells in a Transwell system designed to monitor cell movement through fibroblast generated ECM. After a 24-h period in reduced serum conditions, cell counts showed that the peroxidase induced 3D matrices of MPO and EPO significantly increased $4 \mathrm{~T} 1$ invasion $\sim 1.5$-fold $(\mathrm{P}<0.001)$ compared to vehicle control, a response similar to that seen by the positive control, AA (Fig. 5C).

Peroxidases exhibit direct effects on $4 T 1$ tumour cells. To explore further the role of increased peroxidase presence within the tumour microenvironment, we investigated the proliferation, migration/invasion, and mechanistic aspects of 4T1 tumour cells stimulated with either MPO or EPO. Treatment of $4 \mathrm{~T} 1$ cells with various concentrations of peroxidases had no effect on proliferation after $48 \mathrm{~h}$ when compared to vehicle control (Fig. 6A), while 10\% FBS containing media served as the positive control. However, using the Transwell migration system, the chemo-attractant ability of MPO and EPO significantly increased migration almost 2 -fold $(\mathrm{P}<0.0001)$ and 4 -fold $(\mathrm{P}<0.0001)$ respectively when compared to vehicle control (Fig. 6B). MMP1 gene expression belongs to a subset of genes that are associated with organ specific metastasis to the lungs (28). To investigate the transcription regulation of MMP1, we analyzed peroxidase treated 4T1 tumour cells via RT-PCR. Analysis of mRNA expression showed that MPO and EPO increased MMP1 expression 3- and 2-fold, respectively when compared to vehicle control $(\mathrm{P}<0.05)$.

\section{Discussion}

It is well established that inflammatory cells have commanding effects on tumour development and that both MPO and EPO are found at high levels within the stroma of human breast cancers and breast tumours in mouse models $(4,29)$. The present study is the first to uncover a mechanism linking peroxidases with facilitating tumour growth and metastasis via the regulation of ECM components and angiogenesis. Studies have shown that the depletion of neutrophils can significantly reduce collagendense mammary tumour progression (6) and angiogenesis (30), suggesting that these immune cells and their constituents are key players in tumour formation and metastasis.

Using the murine 4T1 breast cancer model we showed that when injected into the tumour microenvironment, MPO and EPO significantly increased tumour growth and evidence of enhanced metastasis to the lungs. Our unique findings are supported by a recent study showing that specific inhibition of MPO catalytic activity during the early stages of tumour development reduced tumour burden by $50 \%$ in a model of lung carcinogenesis (31). This finding suggests a causative role for MPO during tumorigenesis, and identifies MPO inhibitors as novel therapeutic agents. Furthermore, earlier studies have identified that women with a genetic polymorphism responsible with reduced MPO expression is associated with reduced breast cancer risk $(32,33)$. Taken together, these studies suggest a more sinister role for these inflammatory enzymes.
Our histological analysis of the peroxidase-treated tumours, showed a reduction of necrosis within the necrotic cores inherent of the 4T1 tumours (34). This suggests that MPO and EPO may promote the survival of cancer cells likely via the activation of the stroma compartment since peroxidases had no direct effects on tumour cells themselves.

Peroxidases induced an increase in stromal fibroblasts within the primary tumour and collagen I and VI deposition. Enhanced deposition of collagens and the aberrant changes through the remodeling of ECM composition and function within the tumour microenvironment are crucial for facilitating primary tumour growth and escape (35). Specifically, increased collagen type $I$ is a prognostic factor and is associated with breast cancer recurrence in human breast cancer patients (36), while collagen type VI knockout mice were shown to have reduced primary tumour formation and growth (37). Importantly, the peroxidase effects on primary human mammary fibroblasts in vitro appear to correlate with our histological observations in vivo, as we find that both MPO and EPO regulate fibroblast migration and/or recruitment shown by an increase in $\alpha$-SMA staining within the primary tumours, as wells as increased collagen type I and VI deposition.

Studies have shown that ECM configuration in particular, collagen fiber alignment (27) and tissue stiffness (38) are functionally associated with tumour invasion. In this study, we find that both MPO and EPO, in addition to increasing collagen biosynthesis, alter collagen alignment in a 3D matrix. Our results further show, that the combination of increased collagen biosynthesis with the structural shift in the alignment of collagen fibers in the absence of AA, significantly increased tumour cell adhesion and invasion, however, due to the dramatic ability of peroxidases to stimulate collagen, it may be conceivable that more collagen is sufficient to exert these effects. To accurately recapitulate these effects in vivo, further studies are needed to confirm whether the peroxidaseinduced alignment observed in the present study affects overall tumour stiffness as reported by Acerbi and colleagues (39). Intriguingly, the authors of this study revealed that a correlation between the invasive regions of aggressive human breast cancer and ECM remodeling and stiffening was linked to an increase in innate immune infiltrate. Although, it is widely accepted that elevated collagen deposition coupled with the alteration of linear collagen fibers lead to an increase in ECM stiffness $(40,41)$, further exploration is needed to determine the stromal features that contribute to tumour stiffness.

Aberrant MMP activities within the stromal compartment have a causative role in tumour incidence by affecting tumour cell motility and invasiveness. In the present study, we showed that both MPO and EPO modulate MMP1 and MMP3 gene expression in primary human mammary fibroblasts, while increasing the transcription of MMP1 in 4T1 mammary carcinoma epithelial cells. Elevated MMP1 expression in CAFs has been linked to vital metastatic processes that facilitate angiogenesis, matrix degradation and the conversion of normal resident fibroblasts to activated CAFs (42). In addition, several independent studies reported that the overexpression of MMP3 within the mammary stroma, increases mammary tumour incidence and invasion (43-45), while the overexpression of MMP1 in mammary epithelial cells is associated with 
increased invasion, and metastasis to the lungs, brain and the bone $(28,46-48)$. We have recently shown that MPO and EPO modulate the transcriptional regulation of COX-2 in primary human endothelial cells (15). Here, we show that peroxidases also regulated COX-2 gene expression in primary human fibroblasts. However, no significant effect was found in murine 4T1 carcinoma cells (data not shown). Based on the increased expression in a large portion of breast carcinomas, the activation of COX-2 and MMPs have been implicated to have a significant role in accelerating breast tumorigenesis and necessary for the initiation of metastasis, particularly to the lungs (49). Emerging evidence reveals that stromal COX-2 expression can also function as a mediator of tumour progression $(50,51)$.

In summary, our findings demonstrate for the first time that the peroxidase enzymes MPO and EPO confer a broader range of action than previously thought and exhibit potent pro-tumorigenic effects in the tumour milieu altering matrix composition and function, angiogenesis and invasion. Taken together with our previous reported observations, we propose that both MPO and EPO are causatively involved in breast cancer progression and identify them as potential therapeutic targets whereby specific novel inhibitors may reduce tumour growth and limit the occurrence of metastasis.

\section{Acknowledgements}

We thank Matthew Iasiello for his excellent technical assistance. The present study was supported in part by The Hospital Research Foundation and the National Health and Medical Research Council (Career Development Fellowship/627015; Project Grant/1050694).

\section{References}

1. Mantovani A, Allavena P, Sica A and Balkwill F: Cancer-related inflammation. Nature 454: 436-444, 2008.

2. Hanahan D and Weinberg RA: Hallmarks of cancer: The next generation. Cell 144: 646-674, 2011.

3. Galdiero MR, Bonavita E, Barajon I, Garlanda C, Mantovani A and Jaillon S: Tumor associated macrophages and neutrophils in cancer. Immunobiology 218: 1402-1410, 2013.

4. Samoszuk MK, Nguyen V, Gluzman I and Pham JH: Occult deposition of eosinophil peroxidase in a subset of human breast carcinomas. Am J Pathol 148: 701-706, 1996.

5. Chen J, Deng Q, Pan Y, He B, Ying H, Sun H, Liu X and Wang S: Prognostic value of neutrophil-to-lymphocyte ratio in breast cancer. FEBS Open Bio 5: 502-507, 2015.

6. García-Mendoza MG, Inman DR, Ponik SM, Jeffery JJ, Sheerar DS, Van Doorn RR and Keely PJ: Neutrophils drive accelerated tumor progression in the collagen-dense mammary tumor microenvironment. Breast Cancer Res 18: 49, 2016.

7. Swierczak A, Mouchemore KA, Hamilton JA and Anderson RL: Neutrophils: Important contributors to tumor progression and metastasis. Cancer Metastasis Rev 34: 735-751, 2015.

8. Wculek SK and Malanchi I: Neutrophils support lung colonization of metastasis-initiating breast cancer cells. Nature 528: 413-417, 2015.

9. Tabariès S, Ouellet V, Hsu BE, Annis MG, Rose AA, Meunier L, Carmona E, Tam CE, Mes-Masson AM and Siegel PM: Granulocytic immune infiltrates are essential for the efficient formation of breast cancer liver metastases. Breast Cancer Res 17: 45, 2015.

10. Stockmann C, Schadendorf D, Klose R and Helfrich I: The impact of the immune system on tumor: Angiogenesis and vascular remodeling. Front Oncol 4: 69, 2014.

11. Samoszuk M, Lin F, Rim P and Strathearn G: New marker for blood vessels in human ovarian and endometrial cancers. Clin Cancer Res 2: 1867-1871, 1996.
12. Cormier SA, Taranova AG, Bedient C, Nguyen T, Protheroe C, Pero R, Dimina D, Ochkur SI, O'Neill K, Colbert D, et al: Pivotal Advance: Eosinophil infiltration of solid tumors is an early and persistent inflammatory host response. J Leukoc Biol 79: 1131-1139, 2006

13. Ambrosone CB, Barlow WE, Reynolds W, Livingston RB, Yeh IT, Choi JY, Davis W, Rae JM, Tang L, Hutchins LR, et al: Myeloperoxidase genotypes and enhanced efficacy of chemotherapy for early-stage breast cancer in SWOG-8897. J Clin Oncol 27: 4973-4979, 2009.

14. DeNichilo MO, Panagopoulos V, Rayner TE, Borowicz RA, Greenwood JE and Evdokiou A: Peroxidase enzymes regulate collagen extracellular matrix biosynthesis. Am J Pathol 185: 1372-1384, 2015

15. Panagopoulos V, Zinonos I, Leach DA, Hay SJ, Liapis V, Zysk A, Ingman WV, DeNichilo MO and Evdokiou A: Uncovering a new role for peroxidase enzymes as drivers of angiogenesis. Int $\mathrm{J}$ Biochem Cell Biol 68: 128-138, 2015.

16. Hanahan D and Coussens LM: Accessories to the crime: Functions of cells recruited to the tumor microenvironment. Cancer Cell 21: 309-322, 2012.

17. Keskin D, Kim J, Cooke VG, Wu CC, Sugimoto H, Gu C, De Palma M, Kalluri R and LeBleu VS: Targeting vascular pericytes in hypoxic tumors increases lung metastasis via angiopoietin-2. Cell Rep 10: 1066-1081, 2015.

18. Rangan GK and Tesch GH: Quantification of renal pathology by image analysis. Nephrology (Carlton) 12: 553-558, 2007.

19. Castelló-Cros R and Cukierman E: Stromagenesis during tumorigenesis: Characterization of tumor-associated fibroblasts and stroma-derived 3D matrices. Methods Mol Biol 522: 275-305, 2009.

20. Kenny HA, Krausz T, Yamada SD and Lengyel E: Use of a novel $3 \mathrm{D}$ culture model to elucidate the role of mesothelial cells, fibroblasts and extra-cellular matrices on adhesion and invasion of ovarian cancer cells to the omentum. Int J Cancer 121: 1463-1472, 2007.

21. Fisher KE, Pop A, Koh W, Anthis NJ, Saunders WB and Davis GE: Tumor cell invasion of collagen matrices requires coordinate lipid agonist-induced G-protein and membrane-type matrix metalloproteinase-1-dependent signaling. Mol Cancer 5: 69, 2006.

22. Villa JC, Chiu D, Brandes AH, Escorcia FE, Villa $\mathrm{CH}$, Maguire WF, Hu CJ, de Stanchina E, Simon MC, Sisodia SS, et al: Nontranscriptional role of Hif- $1 \alpha$ in activation of $\gamma$-secretase and notch signaling in breast cancer. Cell Rep 8: 1077-1092, 2014.

23. Leach DA, Need EF, Trotta AP, Grubisha MJ, DeFranco DB and Buchanan G: Hic-5 influences genomic and non-genomic actions of the androgen receptor in prostate myofibroblasts. Mol Cell Endocrinol 384: 185-199, 2014.

24. Pulaski BA and Ostrand-Rosenberg S: Reduction of established spontaneous mammary carcinoma metastases following immunotherapy with major histocompatibility complex class II and B7.1 cell-based tumor vaccines. Cancer Res 58: 1486-1493, 1998.

25. Orimo A, Gupta PB, Sgroi DC, Arenzana-Seisdedos F, Delaunay T, Naeem R, Carey VJ, Richardson AL and Weinberg RA: Stromal fibroblasts present in invasive human breast carcinomas promote tumor growth and angiogenesis through elevated SDF-1/CXCL12 secretion. Cell 121: 335-348, 2005.

26. Cirri P and Chiarugi P: Cancer associated fibroblasts: The dark side of the coin. Am J Cancer Res 1: 482-497, 2011.

27. Provenzano PP, Eliceiri KW, Campbell JM, Inman DR, White JG and Keely PJ: Collagen reorganization at the tumor-stromal interface facilitates local invasion. BMC Med 4: 38, 2006.

28. Minn AJ, Gupta GP, Siegel PM, Bos PD, Shu W, Giri DD, Viale A, Olshen AB, Gerald WL and Massagué J: Genes that mediate breast cancer metastasis to lung. Nature 436: 518-524, 2005.

29. Zhang N, Francis KP, Prakash A and Ansaldi D: Enhanced detection of myeloperoxidase activity in deep tissues through luminescent excitation of near-infrared nanoparticles. Nat Med 19: 500-505, 2013.

30. Nozawa $\mathrm{H}$, Chiu $\mathrm{C}$ and Hanahan D: Infiltrating neutrophils mediate the initial angiogenic switch in a mouse model of multistage carcinogenesis. Proc Natl Acad Sci USA 103: 12493-12498, 2006.

31. Rymaszewski AL, Tate E, Yimbesalu JP, Gelman AE, Jarzembowski JA, Zhang H, Pritchard KA Jr and Vikis HG: The role of neutrophil myeloperoxidase in models of lung tumor development. Cancers (Basel) 6: 1111-1127, 2014. 
32. Ahn J, Gammon MD, Santella RM, Gaudet MM, Britton JA, Teitelbaum SL, Terry MB, Neugut AI, Josephy PD and Ambrosone CB: Myeloperoxidase genotype, fruit and vegetable consumption, and breast cancer risk. Cancer Res 64: 7634-7639, 2004.

33. Pabalan N, Jarjanazi H, Sung L, Li H and Ozcelik H: Menopausal status modifies breast cancer risk associated with the myeloperoxidase (MPO) G463A polymorphism in Caucasian women: A meta-analysis. PLoS One 7: e32389, 2012.

34. Serganova I, Rizwan A, Ni X, Thakur SB, Vider J, Russell J, Blasberg R and Koutcher JA: Metabolic imaging: A link between lactate dehydrogenase A, lactate, and tumor phenotype. Clin Cancer Res 17: 6250-6261, 2011.

35. Cox TR and Erler JT: Remodeling and homeostasis of the extracellular matrix: Implications for fibrotic diseases and cancer. Dis Model Mech 4: 165-178, 2011.

36. van 't Veer LJ, Dai H, van de Vijver MJ, He YD, Hart AA, Mao M, Peterse HL, van der Kooy K, Marton MJ, Witteveen AT, et al: Gene expression profiling predicts clinical outcome of breast cancer. Nature 415: 530-536, 2002.

37. Iyengar P, Espina V, Williams TW, Lin Y, Berry D, Jelicks LA Lee H, Temple K, Graves R, Pollard J, et al: Adipocyte-derived collagen VI affects early mammary tumor progression in vivo, demonstrating a critical interaction in the tumor/stroma microenvironment. J Clin Invest 115: 1163-1176, 2005.

38. Paszek MJ, Zahir N, Johnson KR, Lakins JN, Rozenberg GI, Gefen A, Reinhart-King CA, Margulies SS, Dembo M, Boettiger D, et al: Tensional homeostasis and the malignant phenotype. Cancer Cell 8: 241-254, 2005.

39. Acerbi I, Cassereau L, Dean I, Shi Q, Au A, Park C, Chen YY, Liphardt J, Hwang ES and Weaver VM: Human breast cancer invasion and aggression correlates with ECM stiffening and immune cell infiltration. Integr Biol 7: 1120-1134, 2015.

40. Riching KM, Cox BL, Salick MR, Pehlke C, Riching AS, Ponik SM, Bass BR, Crone WC, Jiang Y, Weaver AM, et al: 3D collagen alignment limits protrusions to enhance breast cancer cell persistence. Biophys J 107: 2546-2558, 2014.

41. Egeblad M, Rasch MG and Weaver VM: Dynamic interplay between the collagen scaffold and tumor evolution. Curr Opin Cell Biol 22: 697-706, 2010.
42. Eck SM, Côté AL, Winkelman WD and Brinckerhoff CE: CXCR4 and matrix metalloproteinase- 1 are elevated in breast carcinoma-associated fibroblasts and in normal mammary fibroblasts exposed to factors secreted by breast cancer cells. Mol Cancer Res 7: 1033-1044, 2009.

43. Sternlicht MD, Safarians S, Rivera SP and Barsky SH: Characterizations of the extracellular matrix and proteinase inhibitor content of human myoepithelial tumors. Lab Invest 74: 781-796, 1996

44. Sympson CJ, Bissell MJ and Werb Z: Mammary gland tumor formation in transgenic mice overexpressing stromelysin-1. Semin Cancer Biol 6: 159-163, 1995.

45. Holliday DL, Hughes S, Shaw JA, Walker RA and Jones JL: Intrinsic genetic characteristics determine tumor-modifying capacity of fibroblasts: Matrix metalloproteinase-3 5A/5A genotype enhances breast cancer cell invasion. Breast Cancer Res 9: R67, 2007.

46. Egeblad M and Werb Z: New functions for the matrix metalloproteinases in cancer progression. Nat Rev Cancer 2: 161-174, 2002.

47. Liu H, Kato Y, Erzinger SA, Kiriakova GM, Qian Y, Palmieri D, Steeg PS and Price JE: The role of MMP-1 in breast cancer growth and metastasis to the brain in a xenograft model. BMC Cancer 12: 583, 2012.

48. Kang Y, Siegel PM, Shu W, Drobnjak M, Kakonen SM, CordónCardo C, Guise TA and Massagué J: A multigenic program mediating breast cancer metastasis to bone. Cancer Cell 3: 537-549, 2003.

49. Gupta GP, Nguyen DX, Chiang AC, Bos PD, Kim JY, Nadal C, Gomis RR, Manova-Todorova K and Massagué J: Mediators of vascular remodelling co-opted for sequential steps in lung metastasis. Nature 446: 765-770, 2007.

50. Erez N, Truitt M, Olson P, Arron ST and Hanahan D: Cancerassociated fibroblasts are activated in incipient neoplasia to orchestrate tumor-promoting inflammation in an NF-kappaBdependent manner. Cancer Cell 17: 135-147, 2010.

51. Park SW, Kim HS, Choi MS, Jeong WJ, Heo DS, Kim KH and Sung MW: The effects of the stromal cell-derived cyclooxygenase- 2 metabolite prostaglandin E2 on the proliferation of colon cancer cells. J Pharmacol Exp Ther 336: 516-523, 2011 